\title{
CALCULATIONS FOR HIGH-ENERGY RADIATIONS *
}

\author{
J.E. TURNER **, H.A. WRIGHT ${ }^{* *}$, and R.N. HAMM ** \\ (Manuscrit rę̧u le 19 juin 1974)
}

\begin{abstract}
The theoretical high-energy dosimetry program, developed over the past decade in the Health Physics Division of the Oak Ridge National Laboratory, is described briefly. Some selected results are presented to illustrate the calculational capabilities that exist. These examples include depth-dose curves for broad, parallel beams of $2 \mathrm{GeV}$ protons and $\mathrm{I} \mathrm{GeV}$ neutrons incident laterally on a tissue slab and ${ }^{11} \mathrm{C}$ activation calculated as a function of depth in a water phantom exposed to a beam of $400 \mathrm{MeV}$ neutrons. Because of their planned use in cancer radiotherapy research, pions are under extensive study. The Monte Carlo computer code, PION-I, was developed to calculate the penetration of pions in materials (containing $H, C, N$, and $O$ ) exposed to pion beams. Good agreement is found between calculated and measured curves in water. Calculated L.E.T. distributions at various depths are shown. The pattern of energy deposition around pion capture sites is analyzed. T-I kidney cell survival levels are predicted for pion beams based on data of Todd and on the survival model of Katz.
\end{abstract}

\section{MONTE-CARLO CALCULATIONS OF PARTICLE PENETRATION}

The calculational programs of the Health Physics Division at the Oak Ridge National Laboratory utilize Monte Carlo methods, which have been exployed extensively by many investigators. Descriptions of such calculations, which simulate the passage of radiation through matter, are given in the literature [r]. The case histories of a large number of incident particles and the secondary particles they produce are generated by a computer. The computer selects specific events (e.g., particle scattering, absorption, or escape from the target) and their locations for each particle based on the probabilities, or differential cross sections, for their occurrence. In principle, measured values of the cross sections can be used, so that the computer calculations simulate actual events as they occur statistically in nature. In practice, however, differential cross sections are often not known in detail.

* Work supported by the U.S. Atomic Energy Commission and the National Science Foundation under contract with Union Carbide Corporation.

** Health Physics Division, Oak Ridge National Laboratory, Oak Ridge, TN 37830 , USA.

vOL. $9-\mathrm{N}^{\circ} 2$ 


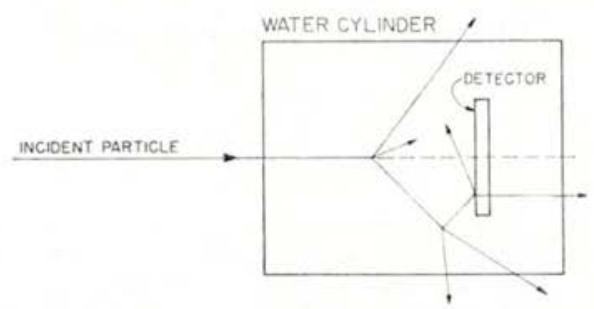

Fig. 1. - Illustration of particle track histories generated in a Monte Carlo calculation.

Figure $\mathrm{I}$ illustrates the track of a particle incident from the left on a water cylinder. The particle experiences a nuclear collision, which produces secondary products that, in turn, may produce other particles. If the dose is desired over a given volume, such as that designated as the detector, then a record is kept of the energy deposited by all particles that enter, or originate in, that volume. Typically, in the calculations reported below, ro, 000 incident particles are used and the dose is calculated in an array of detector volumes that span the target. Since the calculations provide complete microscopic detail of all events, additional quantities can be compiled, such as L.E.T. distributions and other parameters for use in conjunction with biological response models.

The Health Physics Division has developed computer codes for transporting photons, electrons, muons, pions, neutrons, protons, alpha particles and other light nuclei. A few examples follow.

\section{HIGH-ENERGY NUCLEONS}

Calculations have been performed for energies up to $2 \mathrm{GeV}$ for beams of protons and neutrons incident perpendicularly on an infinite soft-tissue slab of thickness $30 \mathrm{~cm} \mathrm{[2].} \mathrm{Some} \mathrm{results} \mathrm{are} \mathrm{presented} \mathrm{in} \mathrm{figures} 2$ and 3 . Dose contributions from various particle types, as well as the total absorbed dose and dose equivalent [3], are shown as functions of depth in the slab. Such computations show the manner in which the primary-particle fluence decreases with depth. The increase in secondary-particle contributions to the dose and the buildup of the total dose within the slab are obtained in detail. 


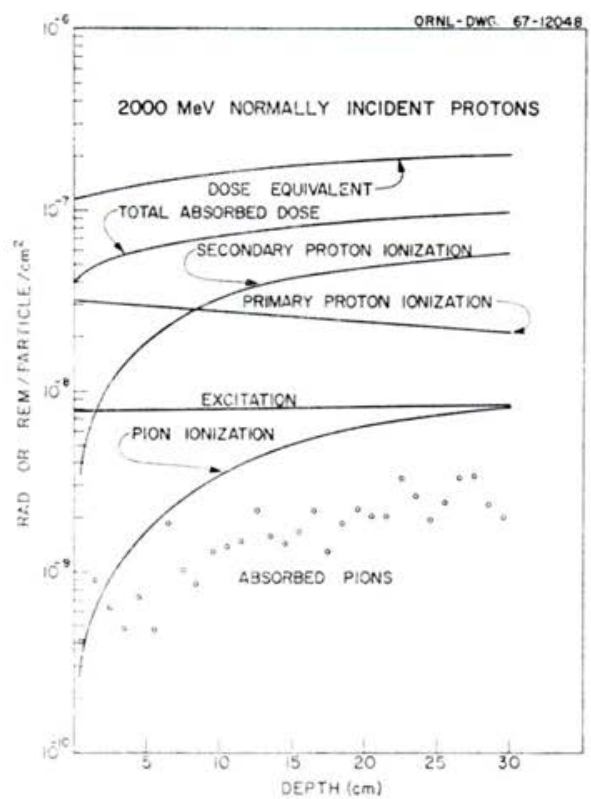

Fig. 2. - Absorbed dose and dose equivalent in a $30 \mathrm{~cm}$ tissue slab irradiated perpendicularly by a broad beam of $2 \mathrm{GeV}$ protons. Also shown are the doses resulting from secondary protons, excited nuclei, pion ionization, and pion absorption.

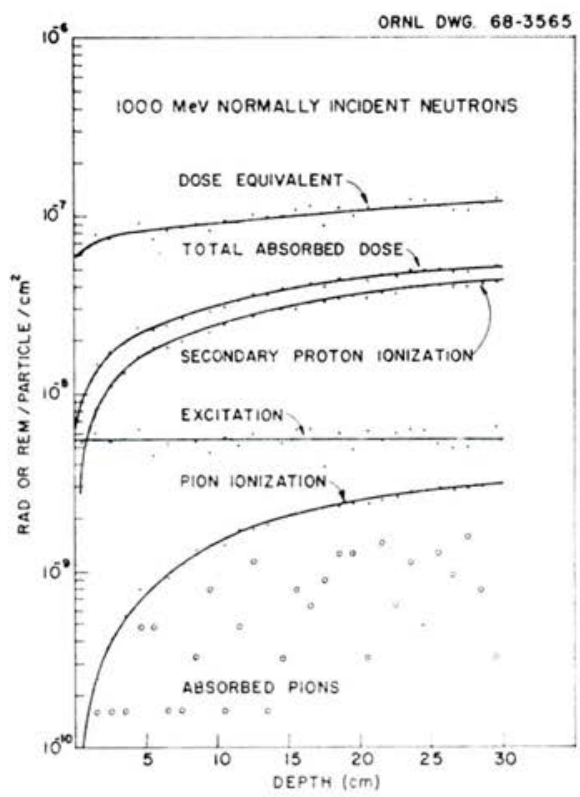

FIG. 3. - Absorbed dose and dose equivalent in a $30 \mathrm{~cm}$ tissue slab irradiated perpendicularly by a broad beam of $\mathrm{I} \mathrm{GeV}$ neutrons. Also shown are the doses resulting from secondary protons, excited nuclei, pion ionization, and pion absorption. Dots show scatter of calculated data.

The computer codes have various applications. For example, they can be used to analyse ${ }^{11} \mathrm{C}$ activation measurements made in the vicinity of high-energy accelerators. This radionuclide is produced by nucleons with energies above the threshold of $20 \mathrm{MeV}$ by the reactions ${ }^{12} \mathrm{C}(n, 2 n){ }^{11} \mathrm{C}$ and ${ }^{12} \mathrm{C}(p, n p)^{11} \mathrm{C}$. Figure 4 illustrates a comparison of calculated and experimentally measured values of ${ }^{11} \mathrm{C}$ activation as functions of depth in a polyethylene absorber exposed to a beam of $400 \mathrm{MeV}$ neutrons. The ordinate shows the activation in arbitrary units. The calculations are, of course, absolute and provide a basis for converting measured ${ }^{11} \mathrm{C}$ activation to neutron fluence.

\section{PION DOSIMETRY}

Pions, which have a rest mass of $140 \mathrm{MeV}$, are produced in collisions of particles with energies of several hundred $\mathrm{MeV}$ or greater. Figures 2 and 3 show the dose due to pions produced in tissue by $2 \mathrm{GeV}$ protons and $\mathrm{I} \mathrm{GeV}$ neutrons. Although the pions themselves may have low kinetic energy, their 


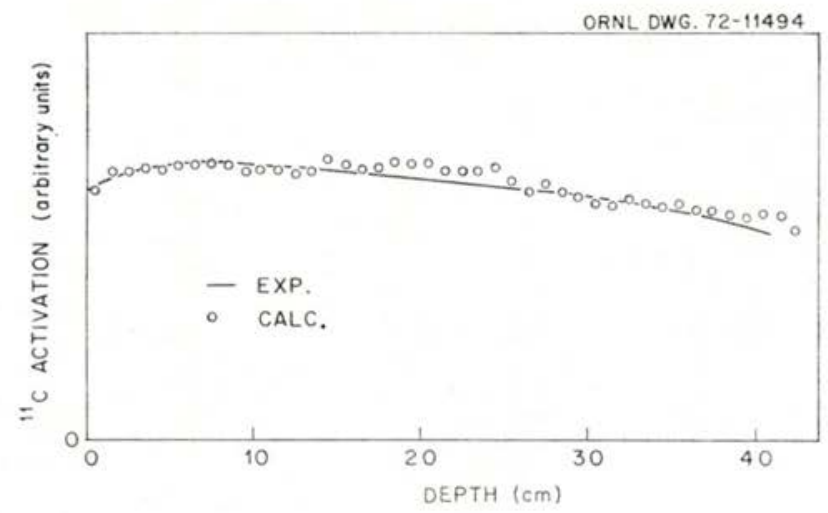

FIG. 4. - Comparison of calculated and experimentally measured ${ }^{11} \mathrm{C}$ activation as functions of depth in a polyethylene absorber exposed to a beam of $400 \mathrm{MeV}$ neutrons.

treatment is an integral part of the Division's high-energy dosimetry program. Interest in pion dosimetry has intensified over the past several years, because of their planned use in radiotherapy. Biomedical pion irradiation facilities now under construction in the United States, Canada, and Switzerland will have experimental beams available within the next year.

The Monte Carlo computer code, PION-I [4, 5], was developed over the past several years for pion studies. The code takes into account pion slowing down (stopping power), pion-nucleus absorption and inelastic scattering, charge

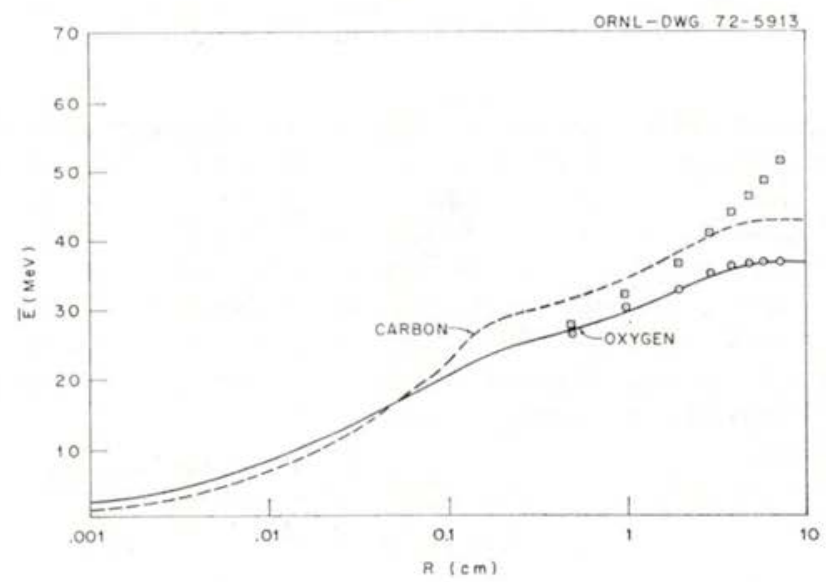

FIG. 5. - Energy deposition in water volumes surrounding site of $\pi^{-}$capture by oxygen and carbon. Solid an dashed curves, calculated for spheres of radius R, show energy deposited by charged particles produced directly from $\pi^{-}$capture. Circles show same for oxygen, calculated for cylinders of radius $R$ and length $2 R$. Squares show the addition of neutrons produced by capture in oxygen. 
exchange, elastic scattering with hydrogen, multiple coulomb scattering, and range straggling. The muon and electron contamination found in typical pion beams can also be handled in PION-I.

The distinguishing feature of a negative pion, which makes it of particular interest for possible radiotherapy, is its absorption by an atomic nucleus at the end of its path. The stopped negative pion is attracted to the positively charged nucleus, which captures and absorbs it. In soft tissue, for example, a stopped negative pion is absorbed by a nucleus of oxygen, carbon, or nitrogen. The pion disappears and gives its rest energy of $140 \mathrm{MeV}$ to the nucleus, which then explodes as a result of having this excess energy. An event of his type is called a star. The heavy nuclear fragments from a star deposit their energy at high L.E.T. within a few millimeters of the capture site.

Figure s shows an analysis of the average energy deposited in water spheres and cylinders around sites where a negative pion is captured by oxygen and carbon nuclei [6]. These calculations indicate that so \% of the charged-particle energy is deposited within a distance of $0.1 \mathrm{~cm}$ from the site and $90 \%$ within $0.2 \mathrm{~cm}$. Figure 6 shows the energy deposited at an L.E.T. above $170 \mathrm{MeV} / \mathrm{cm}$. Practically all particles with initial L.E.T. in this range are absorbed within about $0.2 \mathrm{~cm}$. The results indicate that, on the average, $30 \mathrm{MeV}$ of energy is deposited in a sphere of radius $\mathrm{I} \mathrm{cm}$ surrounding the oxygen capture site and $35 \mathrm{MeV}$ around the carbon site.

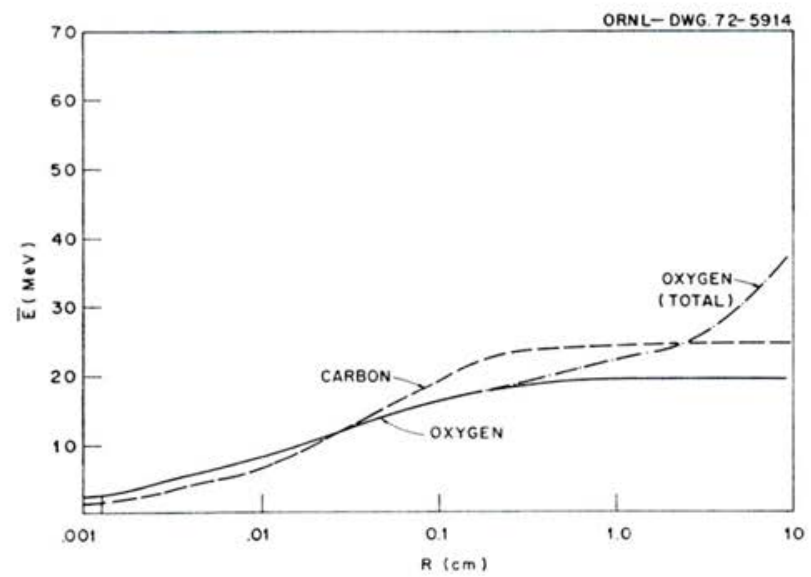

FIG. 6. - Energy deposition in water spheres by high L.E.T. ( $170 \mathrm{MeV} \mathrm{cm}^{-1}$ ) particles produced directly from $\pi^{-}$capture by oxygen (solid curve) and carbon (dashed curve). Added contribution of high L.E.T. recoils from neutrons produced in capture by oxygen is also shown (total).

While direct experimental measurements are not available to verify the predictions of figures $s$ and 6, there are other measurements with pion beams to compare with calculations. The open circles in figure 7 show values of the absorbed dose measured with a $3 \mathrm{~cm}$ radius cylindrical detector placed at various

vOL. $9-\mathrm{N}^{\mathrm{O}}{ }_{2}$ 
depths in a water phantom exposed to a negative pion beam at C.E.R.N. The dose, calculated with an early version of PION-I, is also shown, together with the cumulative contributions from various sources as found in the computations. The density of particles in the beam in the calculations was the same as that inferred from measurements on a film placed in the path of the experimental beam. The fit was obtained by assuming that the mean momentum of particles in the beam was $176 \mathrm{MeV} / \mathrm{c}$ and the momentum spread, assumed Gaussian, was $2 \%$ (i.e., one-half of the particles had momentum within $\pm 2 \%$ of the mean). About is $\%$ of the incident pions interact in flight; the other $85 \%$ come to rest at depths around $20 \mathrm{~cm}$. The small peak at about $28 \mathrm{~cm}$ is due to muon contamination in the beam, the muon dose being shown by the portion of the dose labeled $\mu_{c}^{-}$. Beyond $30 \mathrm{~cm}$ the dose is due mostly to electron contamination in the beam, as indicated by the portion labeled $\mathrm{e}^{-}$. The dose deposited as a result of pion ionization is indicated by $\pi^{-}$. The dose from heavy particles (atomic mass number $>1$ ) and protons is shown by $b+p$. This contribution gets very large in the stopping region, where pion stars occur. The dose from neutrons is shown by the cross-hatched region.

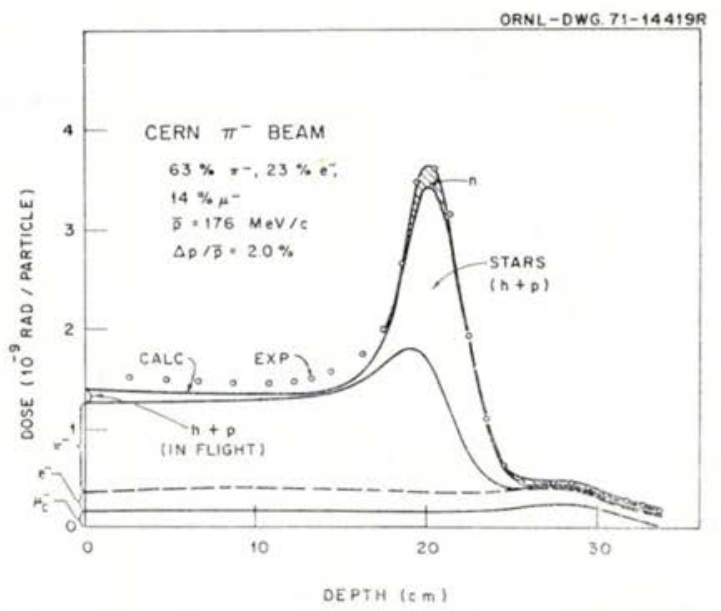

Fig. 7. - Cumulative plot of various contributions to dose calculated for C.E.R.N. pion beam. Experimental values of the total dose are shown by open circles. Curves are explained in the text.

The agreement between calculated and experimentally measured total dose curves is satisfactory. Doubtlessly, even better agreement could be obtained, if warranted, by further adjusting the values of the beam parameters in the computations. As with the ${ }^{11} \mathrm{C}$ activation example, the calculations in figure 7 are absolute and provide a basis for converting pion fluence to dose. 


\section{L.E.T. DISTRIBUTIONS}

Knowledge of L.E.T. distributions is often desired for the comparison and interpretation of radiobiological experiments and, in radiation protection work, for assigning quality factors to estimate dose equivalent. As an example, calculations were made for a uniform, circular negative pion beam of radius $3 \mathrm{~cm}$, the momentum distribution being Gaussian with a mean of $175 \mathrm{MeV} / \mathrm{c}$ and a spread of $2 \%$ [7]. The depth-dose curve is similar to that in figure 7 . The curve is characterized by a flat, or plateau, region to a depth of about $1 \mathrm{~s} \mathrm{~cm}$. Thereafter, the dose rises sharply to a peak between 20 and $21 \mathrm{~cm}$ and falls off beyond. The L.E.T. distribution changes markedly between the plateau and peak regions, because the physical processes that contribute to dose change. The histograms in figure 8 show the percentages of dose in different L.E.T. intervals, averaged between the depths s to $6 \mathrm{~cm}$ and 20.4 to $20.6 \mathrm{~cm}$. The calculations show that $90 \%$ of the dose in the plateau region is deposited with an L.E.T. less than 35 $\mathrm{MeV} / \mathrm{cm}$. The L.E.T. distribution through the plateau begins to change at a depth of about $17.5 \mathrm{~cm}$. In the peak region the doses in the intervals above 35 $\mathrm{MeV} / \mathrm{cm}$ are from four to eight times greater than in the plateau.

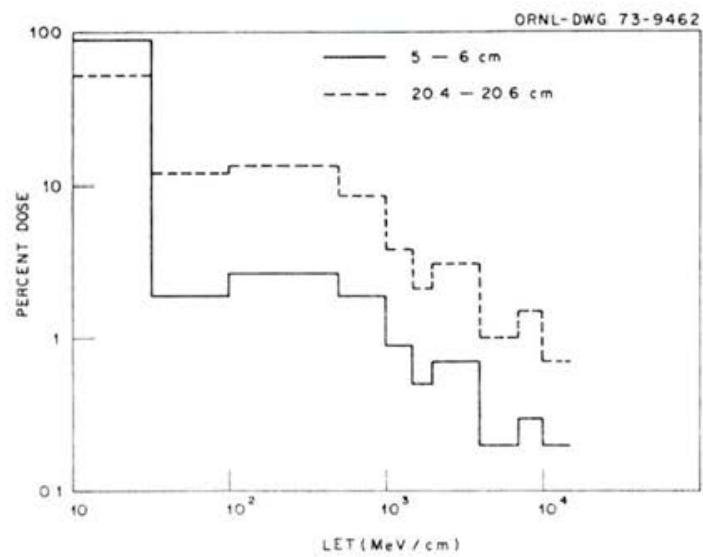

FIG. 8. - Dose distributions as functions of L.E.T. in plateau (solid curve, $5-6 \mathrm{~cm}$ ) and peak (dashed curve, $20.4-20.6 \mathrm{~cm}$ ) regions.

\section{s. PREDICTIONS OF CELL SURVIVAL}

TODD has summarized survival data for T-I human kidney cells exposed to different ions [8]. He presents a graph, showing measured R.B.E. values versus the L.E.T. of the radiation at various levels of survival. These values have been used as weighting factors for the absorbed dose in various L.E.T. intervals to compute an " effectivedose" as a function of depth for a pure pion beam [9].

vOL. $9-\mathrm{N}_{2}$ 


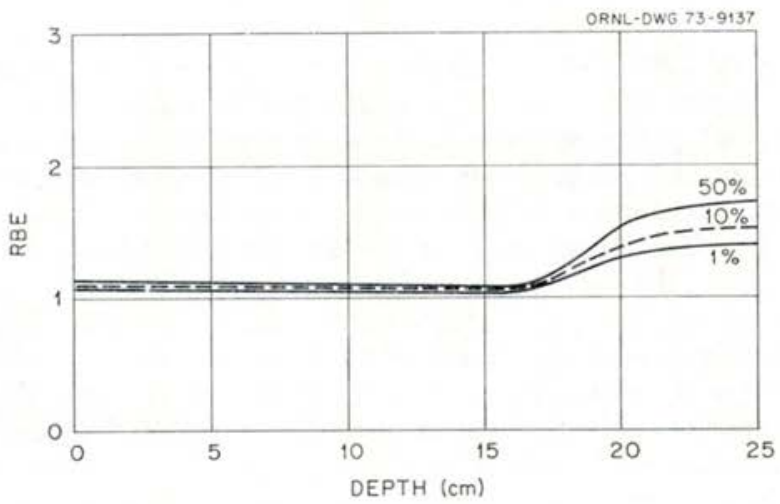

Fig. 9. - Estimated R.B.E. as a function of depth for $50 \%$, 10 \%, and I \% survival of T-I human kidney cells. (Based on data of Ref. 8).

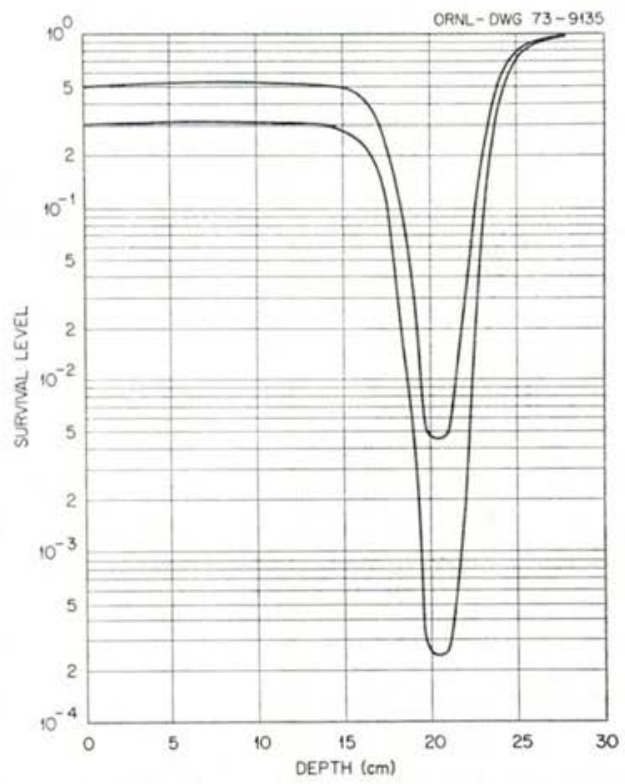

FIG. I0. - Estimated survival levels for T-1 human kidney cells at different depths when survival at surface is $50 \%$ and $30 \%$ (calculated with Katz's model, Ref. ro). 
(The beam was the same as that for figure 7, except that the mean momentum was $175 \mathrm{MeV} / \mathrm{c}$ and muons and electrons were excluded. The doses were averaged over cylinders of $3 \mathrm{~cm}$ radius along the beam axis.) Dividing the weighted dose at a given survival level by the absorbed dose gives an estimate of the R.B.E. as a function of depth for that level. Some results are shown in figure 9. They indicate that the pion R.B.E. values are somewhat greater than unity in the plateau and may reach maximum values of $1.4,1.5$, and 1.7 at depths slightly beyond that at which the peak dose occurs.

Survival calculations for pions have also been made with Katz's mode [ro]. For the beam just described survival curves were calculated as functions of depth for pion doses that give $50 \%$ and $30 \%$ survival at the surface [9]. The predictions are shown in figure ro. The survival probability rises slightly over the first 7 or $8 \mathrm{~cm}$ of depth, where the absorbed dose decreases. The survival level decreases rapidly where the dose begins to peak. The upper curve in figure ro drops two orders of magnitude and the lower curve, three. R.B.E. values calculated with Katz's model for T-I cell survival appear to be $\simeq 50 \%$ higher than those estimated from Todd's work (fig. 9).

\section{SUMMARY}

The work presented here represents only a small sample of the information that can be calculated with the high-energy dosimetry computer programs of the Health Physics Division. Results obtained with some of the computer codes, such as pion depth-dose curves and ${ }^{11} \mathrm{C}$ activation, have been compared directly with experiment. Such comparisons have not revealed any gross discrepancies between calculation and measurement. The physics involved in the programs is thought to be well understood. Individual cross sections used in the Monte Carlo calculations are probably known well enough for reliable computations of averaged quantities, such as doses and L.E.T. distributions. Whereas experimental measurements with high-energy particles are always costly, computer calculations offer a relatively inexpensive way of obtaining information. Such calculations must ultimately be supported, of course, on the basis of actual measurements.

Computed biological predictions of such quantities as pion R.B.E. and cell survival do not warrant the same degree of confidence as physical predictions. Even with a good understanding of the physics of radiation absorption and dose deposition, biological uncertainties of unknown magnitudes enter. Knowledge of the degree of reliability of calculated biological results awaits experiment.

\section{REFERENCES}

[I] For example, see Martin J. Berger, Monte Carlo Calculation of the Penetration and Diffusion of Fast Charged Particles, pp. 135-214 in Metbods in Computational Pbysics, Academic Press, New York (1963).

[2] Wright Harvel A., Anderson V.E., Turner J.E., Neufeld Jacob, Snyder W.S. Health Phys., 16, 13 (1969).

vOL. $9-\mathrm{N}_{2}$ 
[3] The dose equivalent was calculated by using the ICRP/ICRU LET-dependent quality factors (earlier called RBE) given in Healtb Phys., 9, 357 (1963).

[4] WRIGHT H.A. et al., manuscript in preparation.

[s] Turner J.E., Dutrannots J., Wright H.A., Hamm R.N., BaArli J., Sullivan A.H., Berger M.J., Seltzer S.M., Rad. Res., 52, 229 (1972).

[6] Dutrannois J.R., Hamm R.N., Turner J.E., Wright H.A. Phys. Med. Biol., 17, 765 72(19).

[7] Turner J.E., Hamm R.N., Wright H.A. Proceedings of the Fourth Symposium on Microdosimetry, Verbania, Italy, September 1973, Commission of the European Communities, Brussels, Belgium (1974).

[8] Todn Paul. Rad. Res. Suppl., 7, 196 (1967).

[9] Wright H.A., Hamm R.N., Turner J.E. Proceedings of the Third International Congress of the International Radiation Protection Association, Washington, DC, September 1974.

[io] Katz R., Ackerson B., Homayoonfar M., Sharma S.C. Rad. Res., 47, 402 (1971). 